\title{
Resonant Plasmon Response of a Periodically Modulated Two-Dimensional Electron Gas
}

\author{
P. Sznajder ${ }^{a}$, B. Piectka ${ }^{a}$, J. Szczytko $^{a}$, J. Lusakowski $^{a}$ And W. Bardyszewski ${ }^{b}$ \\ ${ }^{a}$ Institute of Experimental Physics, Faculty of Physics, University of Warsaw, Warsaw, Poland \\ ${ }^{b}$ Institute of Theoretical Physics, Faculty of Physics, University of Warsaw, Warsaw, Poland
}

\begin{abstract}
We report the theoretical study of the optical response of a periodically modulated two-dimensional electron gas. The density of states is calculated within the first order of the perturbation theory and the effects of the short-range disorder are explained and discussed. We demonstrate that the magnetic field values corresponding to the characteristic narrowing of the density of states width are given by the zeros of the subsequent Laguerre polynomials. The observed increase of the density of states at the edges are interpreted as van Hove singularities. The broadening effects are shown to modify and smear out the observed effects with increasing temperature above $2 \mathrm{~K}$. The plasmon dispersion relation is discussed in terms of the random phase approximation. Small changes in plasmon dispersion relation related to the periodic modulation were predicted.
\end{abstract}

PACS: 52.25.Mq, 52.40.Db, 73.20.-r, 71.10.Ca, 73.20.Mf

\section{Introduction}

A two-dimensional electron gas (2DEG) can resonantly couple to the electromagnetic radiation of $\mathrm{THz}$ frequencies $[1,2]$. At low temperatures and magnetic fields this system exhibits low energy resonances which include, among others, magnetoplasmons, edge plasmons, magneto-excitons or the Bernstein modes. In this study we concentrate on magnetoplasmons as the basic collective excitations of the $2 \mathrm{DEG}$ at $\mathrm{THz}$ frequencies. The momentum conservation principle forbids the coupling of plasmons to electromagnetic waves at the normal incidence. To overcome this restriction, one may impose a lateral modulation of 2DEG. Such modulation is typically induced by a metallic grid or an interference pattern of the laser light.

Following this idea, we have investigated properties of a 2DEG which is subject to a spatially periodic modulation by an electrostatic potential. This perturbation to the system allows for an efficient coupling of the incident $\mathrm{THz}$ radiation with a $2 \mathrm{D}$ plasmon due to a broken translational symmetry. The mechanism of the coupling can be understood in terms of the Brillouin zone folding. As a result of modulation the plasmon dispersion relation is folding which allows for intersections with a photon dispersion relation. This opens the possibility for a photon-plasmon coupling.

\section{Model}

Considered system was the $2 \mathrm{DEG}$ in a $x y$ plane which was subjected to a uniform perpendicular magnetic field $B$, and a periodically modulated weak potential $V(x)=V_{0} \cos (G x)$, where $2 \pi G^{-1}$ was a period of modulation. The one-electron Hamiltonian of the system in the effective mass approximation regime was as follows:

$$
H=\frac{1}{2 m_{\mathrm{e}}^{*}}(\boldsymbol{p}+e \boldsymbol{A})^{2}+V(x),
$$

where $m_{\mathrm{e}}^{*}$ was the effective mass of an electron in the 2DEG, $\boldsymbol{p}$ was a momentum vector, $e$ was the elementary charge, $\boldsymbol{A}=(0, B x, 0)$ was a vector potential in the Landau gauge. The solution of the unperturbed system (without the $V(x)$ potential) was a set of normalized wave functions

$$
\psi_{n k_{y}}(x, y)=\frac{1}{\sqrt{L_{y}}} \mathrm{e}^{\mathrm{i} k_{y} y} \phi_{n}\left(x+x_{0}\right),
$$

where $L_{y}$ was the length of the system in the $y$ direction, $k_{y}$ was a wave vector in the $y$ direction and $\phi_{n}(x)$ was the normalized $n$-th order harmonic-oscillator function with an equilibrium displacement $x_{0}=l^{2} k_{y}$, where $l=\sqrt{\hbar / e B}$ denotes the magnetic length. The index $n$ corresponds to the $n$-th Landau level (LL). The corresponding eigenvalues are of the form $E_{n}=\hbar \omega_{\mathrm{c}}\left(\frac{1}{2}+n\right)$, where $\omega_{\mathrm{c}}=e B / m_{\mathrm{e}}^{*}$ was the cyclotron frequency. The eigenstates and the corresponding eigenvalues of the full Hamiltonian (1) were evaluated within the first order of the perturbation theory. The eigenenergies $E_{n k_{y}}$ were given by [3]:

$$
E_{n k_{y}}=\hbar \omega_{\mathrm{c}}\left(\frac{1}{2}+n\right)+V_{0} \cos \left(G x_{0}\right) \mathrm{e}^{-u / 2} L_{n}(u),
$$

where $u=G^{2} l^{2} / 2$ and $L_{n}(u)$ denoted the Laguerre polynomial of the $n$-th order. In this approximation the new eigenfunctions $\Psi_{n k_{y}}$ were as follows: 


$$
\Psi_{n k_{y}}=\psi_{n k_{y}}(x, y)+\sum_{n^{\prime} \neq n} \frac{V_{n n^{\prime}}}{E_{n}-E_{n^{\prime}}} \psi_{n^{\prime} k_{y}}(x, y),
$$

where $V_{n n^{\prime}}=\left\langle\psi_{n k_{y}}|V(x)| \psi_{n^{\prime} k_{y}}\right\rangle$ was a matrix element of the operator $V(x)$ in the basis of the $\psi_{n k_{y}}$ states. In this approximation it was assumed that the wave function correction was small in terms of normalization. It means that $\left\langle\Psi_{n k_{y}} \mid \Psi_{n^{\prime} k_{y}^{\prime}}\right\rangle \approx \delta_{n n^{\prime}} \delta_{k_{y} k_{y}^{\prime}}$, which implied $\sum_{n \neq n^{\prime}} \frac{V_{n n^{\prime}}}{E_{n}-E_{n^{\prime}}} \psi_{n^{\prime} k_{y}}(x, y) \approx 0$. This would be useful while deriving the dynamic dielectric function (DDF) within random phase approximation (RPA) for the 2DEG. Equation (3) provides full information about density of states (DOS).

\section{Results and discussion}

The results of numerical calculations are presented in Fig. 1 and Fig. 2. The DOS is broadened by the periodic modulation and the broadening oscillates as function system parameters. The magnetic field value of the minimum of the DOS width is given by [4]:

$$
B_{j}=\frac{2 n \hbar}{\pi e} G\left(j+\frac{3}{4}\right)^{-2},
$$

where $j$ denotes subsequent minima. Important feature is that $j<n$ because of Eq. (5). This approximation is very accurate for large $n$. Similarly, the width of the Landau band at the Fermi level was given by the approximate relation

$$
B_{j}=\frac{\hbar k_{\mathrm{F}}}{\pi e} G\left(j+\frac{3}{4}\right)^{-1} .
$$

Second significant effect of the periodic modulation is the increase of the DOS at the edges of the LL. These are known as the van Hove singularities.

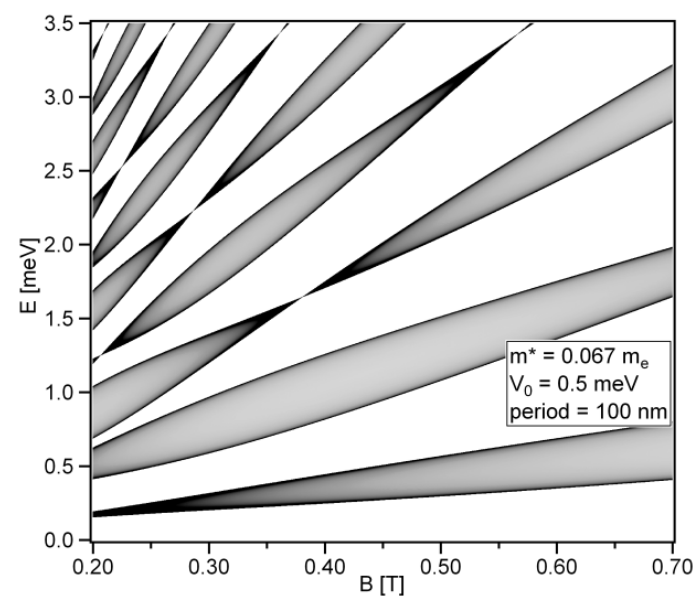

Fig. 1. The DOS as function of magnetic field for the $100 \mathrm{~nm}$ period of the perturbation potential. The minimum value of DOS in the intensity scale is presented in white colour, the maximum in black in arbitrary units.

The oscillations of DOS in magnetic field were observed experimentally by Weiss in Refs. [5, 6] and are known as the Weiss oscillations.

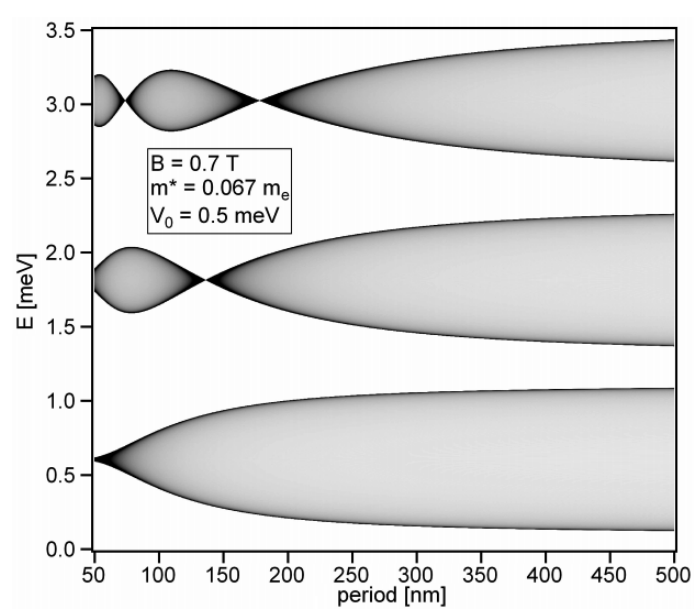

Fig. 2. The evolution of the DOS for different periods of the perturbation potential and for the magnetic field of $0.7 \mathrm{~T}$. The minimum value of DOS in the intensity scale is presented in white colour, the maximum in black in arbitrary units.

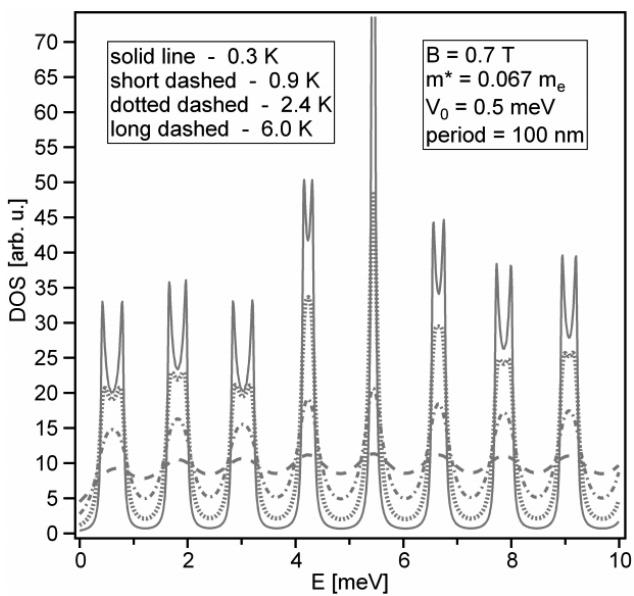

Fig. 3. The Lorentzian broadening of the DOS for four different FWHM parameters $\Gamma=k_{\mathrm{B}} T$, characterized by temperatures as marked in the picture.

The DOS calculated within Eq. (3) of the 2DEG in perpendicular magnetic field with periodic modulation is considered in the ideal case of $T=0 \mathrm{~K}$ and no broadening effects due to disorder. This problem gets more complicated when matched to real experiments. To get more realistic information about the structure of the DOS the disorder is included and the results of our calculations are shown in Fig. 3. We include only short ranged uncorrelated disorder potential caused by dopants and impurities. The resulting DOS is broadened in terms of convolution with Lorentzian curve. This is described by

$$
\rho_{\Gamma}(E)=\int_{-\infty}^{+\infty} \rho(\tilde{E}) \Gamma(E-\tilde{E}) \mathrm{d} \tilde{E},
$$

where $\Gamma$ denotes the Lorentz distribution function. Here $\rho_{\Gamma}$ denotes DOS $\rho$ broadened by the disorder. Obviously 
for high enough level of disorder the van Hove singularities are no more visible. One way of probing the effect of disorder on the density of states is by the photoluminescence (PL) experiments. Unfortunately these measurements provide only convolved information about the DOS. This should be contrasted to transport experiments that probe the DOS at the Fermi level and are dependent mostly on the localized states.

Calculated DOS provides information on the plasmon dispersion relation in the 2DEG modulated by periodic potential. This is crucial for the efficient resonant coupling of the $\mathrm{THz}$ radiation to the $2 \mathrm{DEG}$ system. The plasmon resonances are given by the singularities in the real part of the inverse dielectric function. The dielectric function for the 2DEG in the uniform magnetic field is calculated within the Lindhard formula

$$
\begin{aligned}
& \epsilon_{r}(\omega, \boldsymbol{q})=1+\frac{4 \pi e^{2}}{q^{2}} \frac{2}{\Omega} \\
& \quad \times \sum_{n n^{\prime} k k^{\prime}} \frac{f_{n k}-f_{n^{\prime} k^{\prime}}}{E_{n k}-E_{n^{\prime} k^{\prime}}-\hbar(\omega+\mathrm{i} \eta)}\left|M_{n n^{\prime} k k^{\prime}}\right|^{2},
\end{aligned}
$$

where

$$
\begin{aligned}
& M_{n n^{\prime} k k^{\prime}} \approx\left\langle n k\left|\mathrm{e}^{\mathrm{i} \boldsymbol{q} \boldsymbol{r}}\right| n^{\prime} k^{\prime}\right\rangle \\
& =\left\{\begin{array}{cc}
\delta_{k_{y}^{\prime}, k_{y}-q_{y}} \mathrm{e}^{-\mathrm{i} \Theta-\gamma / 2}\left(\frac{2^{n} n !}{2^{n^{\prime}} n^{\prime} !}\right)^{1 / 2} & \\
\times v^{n^{\prime}-n} L_{n}^{n^{\prime}-n}(\gamma), & n \leq n^{\prime}, \\
\delta_{k_{y}^{\prime}, k_{y}-q_{y}} \mathrm{e}^{-\mathrm{i} \Theta-\gamma / 2}\left(\frac{2^{n} n^{\prime} !}{2^{n^{\prime}} n !}\right)^{1 / 2} & \\
\times\left(-v^{*}\right)^{n-n^{\prime}} L_{n^{\prime}}^{n-n^{\prime}}(\gamma), & n \geq n^{\prime},
\end{array}\right.
\end{aligned}
$$

and $f_{n k}$ is the Fermi-Dirac distribution function. Here $\gamma=l^{2} q^{2} / 2, \Theta=l^{2} q_{x}\left(-k_{y}+q_{y} / 2\right), v=l\left(q_{y}+\mathrm{i} q_{x}\right) / 2$ and $L_{n}^{n^{\prime}}$ is the associated Laguerre polynomial. This is the consequence of using the first order perturbation theory because corrections for the matrix elements $M_{n n^{\prime} k k^{\prime}}$ can be neglected. For simplicity only $q$ in the $x$ direction are considered. This implies the following reduction of the sum in:

$$
\begin{aligned}
& \epsilon_{r}(\omega, q)=1+\frac{4 \pi e^{2}}{q^{2}} \frac{2}{\Omega} \\
& \quad \times \sum_{n n^{\prime} k} \frac{f_{n k}-f_{n^{\prime} k}}{E_{n k}-E_{n^{\prime} k}-\hbar(\omega+\mathrm{i} \eta)}\left|M_{n n^{\prime} k k}\right|^{2} .
\end{aligned}
$$

The above Eq. (9) is slightly different from Eq. (8). The matrix element in the sum is approximately the same as in the absence of the modulating potential, so is the value of the Fermi-Dirac distribution function. The main perturbing term in formula given by Eq. (9) is $E_{n k}-E_{n^{\prime} k}$. If the modulation period is short enough, these differences will be much affected by the perturbation. Hence the plasmon resonance peaks must shift. This should be the main feature observed at very low temperature experiments with short period of modulation. This delicate effects could be observed in the Raman spectroscopy experiments. The difference in the value of the $f_{n k}-f_{n^{\prime} k}$ is only observed in the regime of the liquid helium temperature where this term gets significant around the Fermi level. This means that the dielectric function would not be affected in the regime of energies higher than few $k_{\mathrm{B}} T$, where $k_{\mathrm{B}}$ is the Boltzmann constant. Therefore, at high temperature the effect of the modulation will not be visible in the DOS.

\section{Summary}

The density of states of the periodically modulated 2DEG were calculated. The periodic modulation slightly affects the plasmon dispersion relation in the regime of the first order perturbation theory and the RPA by the modification of the density of states. The width of the density of states oscillates and the strong increase of density is observed at the edges of the Landau bands.

\section{Acknowledgments}

This work was partially supported by a Foundation for Polish Science grant POMOST/2010-1/8 and a Polish Ministry of Science and Higher Education grant N N515 608139 .

\section{References}

[1] M. Dyakonov, M.S. Shur, Phys. Rev. Lett. 71, 2465 (1993).

[2] S.A. Mikhailov, Phys. Rev. B 58, 1517 (1998).

[3] F.M. Peeters, P. Vasilopoulos, Phys. Rev. B 46, 4667 (1992).

[4] M.S. Kushwaha, Surf. Sci. Rep. 41, 123, 363 (2001).

[5] D. Weiss, K. v. Klitzing, K. Ploog, G. Weimann, Europhys. Lett. 8, 179 (1989).

[6] D. Weiss, C. Zhang, R.R. Gerhardts, K. v. Klitzing, G. Weimann, Phys. Rev. B 39, 13020 (1989). 